\title{
Experimental Investigation of
}

Fully Plastic Contact of a Sphere

e-mail: j.jamari@ctw.utwente.nl

\section{J. Schipper}

University of Twente, Surface Technology and Tribology, Faculty of Engineering Technology, Drienerloolaan 5,

Postbus 217,

$7500 \mathrm{AE}$,

Enschede, The Netherlands Against a Hard Flat

In this paper we report the experimental investigation to evaluate the published models for the contact of a deformable sphere against a hard flat in the fully plastic contact regime. A new measurement method has been used to measure the contact area. The behavior of the mean contact pressure and the contact area as a function of the contact load are presented. Substantial differences are found between the measurements and the model predictions. A constant value of the mean contact pressure as the load increases is observed, however, the value is lower than the hardness, as often reported. The contact area is found to be a simple truncation of the sphere by a hard flat.

[DOI: $10.1115 / 1.2164470]$

\section{Introduction}

Depending on the contact situation, in the case when two nominally flat surfaces are pressed against each other, the contacting surface asperities will deform elastic, elastic-plastic, or fully plastic. Greenwood and Williamson [1] defined a plasticity index to predict the degree of plasticity. Plastic contact deformation will be dominant for a plasticity index value greater than 1.0, where in this situation the plasticity will occur even at trivial normal loads. In most of the engineering applications plastic deformation is inevitable.

In the study of friction, wear and lubrication, knowledge about the deformation state of the surface asperities becomes very important. Understanding the relationship between local contact properties and surface topography can lead to the specification of optimized surface topography and manufacturing processes with respect to the functional task of the surface. However, the exact solution for the elastic-plastic contact is difficult to obtain due to the complex variables involved. Significant effort has been put forward by many authors [2-14] to model the elastic-plastic behavior of two bodies in contact.

In 1933, Abbott and Firestone (AF model) [2] introduced the basic plastic contact model, known as the surface microgeometry model. In this model the deformation of a rough surface against a smooth rigid flat is assumed to be equivalent to the truncation of the undeformed rough surface at its intersection with the flat.

The area of contact, $A_{\mathrm{AF}}$, of a sphere in contact with a flat is simply the geometrical intersection of the original profile, represented by a paraboloid, with the flat:

$$
A_{\mathrm{AF}}=2 \pi R \omega
$$

where $R$ is the radius of the sphere and $\omega$ is the contact interference. The mean contact pressure is the flow pressure or the indentation hardness, $H$, and remains constant, therefore the contact load, $P_{\mathrm{AF}}$, is

$$
P_{\mathrm{AF}}=2 \pi R \omega H
$$

Greenwood and Williamson (GW model) [1] developed an asperity contact model for the elastic contact situation. In order to bridge the two extreme models of GW and AF, Chang-EtsionBogy (CEB model) [3] developed an elastic-plastic contact model based on volume conservation of the plastically deforming asperities. The CEB model defined the mean contact pressure in the

Contributed by the Tribology Division of ASME for publication in the JouRnAL OF TRIBOLOGY. Manuscript received June 16, 2005; final manuscript received December 12, 2005. Review conducted by Yeau-Ren Jeng. fully plastic regime by $K H$ where $K$ is the maximum contact pressure factor when initial yield occurs. The contact area, $A_{\mathrm{CEB}}$, and load, $P_{\mathrm{CEB}}$, of the CEB model are expressed by

$$
\begin{gathered}
A_{\mathrm{CEB}}=\pi R \omega\left(2-\frac{\omega_{c-\mathrm{CEB}}}{\omega}\right) \\
P_{\mathrm{CEB}}=\pi R \omega\left(2-\frac{\omega_{c-\mathrm{CEB}}}{\omega}\right) K H
\end{gathered}
$$

In Eqs. (3) and (4), $\omega_{c-\mathrm{CEB}}$ is the critical interference of the onset of plasticity:

$$
\omega_{c-\mathrm{CEB}}=\left(\frac{\pi K H}{2 E}\right)^{2} R
$$

where

$$
\frac{1}{E}=\frac{1-\nu_{1}^{2}}{E_{1}}+\frac{1-\nu_{2}^{2}}{E_{2}}
$$

and $E_{1}, E_{2}, \nu_{1}$, and $\nu_{2}$ are the elastic moduli and the Poisson's ratios of the sphere and the flat, respectively. Horng [4] extended the CEB model to the elliptical contact situation. The elasticplastic or elastoplastic contact regime is not defined by the CEB model; therefore Zhao et al. [5] proposed an elastic-plastic contact model description that incorporates the transition from fully elastic to fully plastic flow. Jeng and Wang [6] extended the model of Zhao to the elliptical contact situation.

Based on finite element analysis (FEA), studies on the contact behavior of two spheres during loading have been reported by Vu-Quoc et al. [7,8], Mesarovic and Fleck [9,10] and Li et al. [11]. A detailed FEA elastic-plastic contact analysis of a sphere and a rigid flat has been performed by Kogut and Etsion [12]. The analysis provided empirical coefficients for the dimensionless relations for contact load, real contact area and mean contact pressure as a function of interference, $\omega$. Due to their nondimensional nature, these relations are applicable to different sphere materials and geometries. However, the analysis is limited up to the onset of the plastic regime. Similar work has been performed by Jackson and Green (JG model) $[13,14]$. The JG model analysis covers the elastic to fully plastic contact regime. This model showed that in the fully plastic contact regime the contact area is larger than that predicted by the AF model hence as a result the mean contact pressure drops with increasing interference. More specifically shown by this model, the contact area and load are dependent 
upon the material properties in the elastic-plastic and the fully plastic regime. In the elastic-plastic and the fully plastic regime the contact area, $A_{\mathrm{JG}}$, is defined as

$$
A_{\mathrm{JG}}^{*}=\omega^{*}\left(\frac{\omega^{*}}{\omega_{t}^{*}}\right)^{B}
$$

where

$$
\begin{aligned}
A_{\mathrm{JG}}^{*} & =\frac{A_{\mathrm{JG}}}{A_{c-\mathrm{JG}}} \\
\omega^{*} & =\frac{\omega}{\omega_{c-\mathrm{JG}}} \\
\omega_{t}^{*} & =1.9
\end{aligned}
$$

and

$$
B=0.14 \exp \left(23 \frac{Y}{E}\right)
$$

and

$$
\begin{gathered}
A_{c-\mathrm{JG}}=\pi^{3}\left(\frac{C Y R}{2 E}\right)^{2} \\
\omega_{c-\mathrm{JG}}=\left(\frac{\pi C Y}{2 E}\right)^{2} R \\
C=1.295 \exp \left(0.736 \nu_{1}\right)
\end{gathered}
$$

$Y$ and $\nu_{1}$ are the yield stress and the Poisson's ratio of the material that yields first, respectively. The contact load, $P_{\mathrm{JG}}$, is formulated by

$$
\begin{aligned}
P_{\mathrm{JG}}^{*}= & {\left[\exp \left(-0.25\left(\omega^{*}\right)^{5 / 12}\right)\right]\left(\omega^{*}\right)^{1.5} } \\
& +\frac{4 H_{G}}{C Y}\left[1-\exp \left(-\frac{1}{25}\left(\omega^{*}\right)^{5 / 9}\right)\right] \omega^{*}
\end{aligned}
$$

where

$$
P_{\mathrm{JG}}^{*}=\frac{P_{\mathrm{JG}}}{P_{c-\mathrm{JG}}}
$$

$$
H_{G}=2.84 Y\left(1-\exp \left\{-0.82\left[\frac{\pi C Y}{2 E} \sqrt{\omega^{*}}\left(\frac{\omega^{*}}{\omega_{t}^{*}}\right)^{B / 2}\right]^{-0.7}\right\}\right)
$$

and

$$
P_{c-\mathrm{JG}}=\frac{4}{3}\left(\frac{R}{E}\right)^{2}\left(\frac{C}{2} \pi Y\right)^{3}
$$

Summarizing, there are several approaches to predict the contact load, contact area, and the mean contact pressure for the contact of a sphere or hemisphere against a hard flat. In order to verify the proposed models experimental investigation is needed. In this paper we present the experimental results of the contact of a deformable sphere against a hard flat for different material properties and radii. A new measuring technique was used to measure the real contact area. These experimental results are compared with the different models discussed to be able to select the best model for a further analysis of the contact between rough surfaces.

\section{Experimental Procedure}

Experiments were performed on a pin-on-disk machine. The flat specimen $(\mathrm{SiC})$ was firmly mounted on a disk and was held statically to apply simple normal loading. The pin of the tester was replaced by a sphere specimen holder. The holder was made from steel and was designed such that the hemispherical form is maintained, as shown in Fig. 1. The maximal load that could be applied to this setup was $600 \mathrm{~N}$.

2.1 Specimens. Copper spheres $(H=1.2 \mathrm{GPa}, E=120 \mathrm{GPa}$, $\nu=0.35$ ) with a diameter of $3 \mathrm{~mm}$ were used as a sphere specimen to perform the experiments. The hard flat specimen used was a $\mathrm{SiC}$ ceramic $(H=28 \mathrm{GPa}, E=430 \mathrm{GPa}, \nu=0.17)$. The copper specimens were as-received without annealing and having a centerline average roughness $R_{a}$ of about $0.15 \mu \mathrm{m}$. The average roughness of the flat specimen was $0.09 \mu \mathrm{m}$.

Most of the proposed asperity contact models assume an elastic perfectly plastic (no-strain hardening) material behavior, therefore the same material properties were used in this experiment. To be sure that there is no strain hardening effect of the copper spheres used, hardness measurements were done before and after a compression test. The Vicker's hardness before compression was $1.15 \mathrm{GPa}$ and after compression at $a / R=0.3$ was $1.2 \mathrm{GPa}$, where

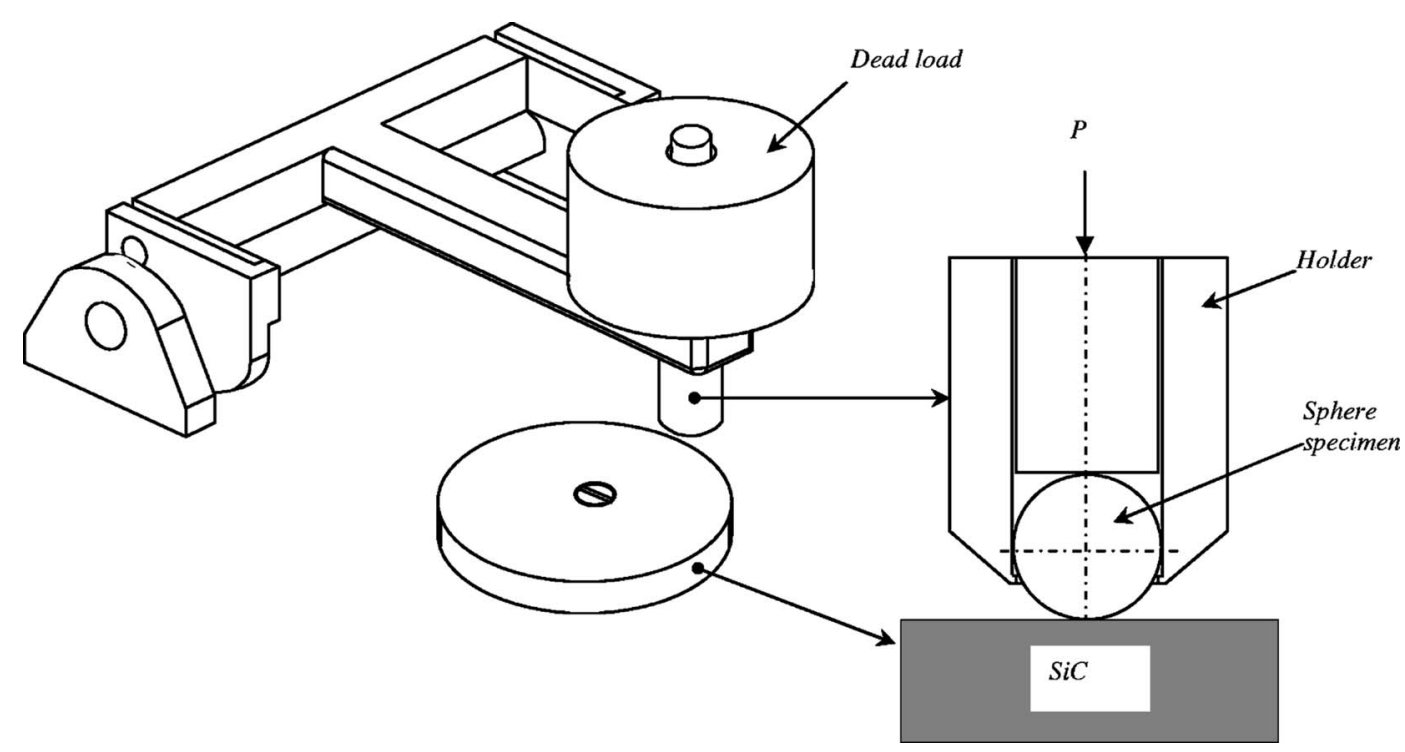

Fig. 1 Setup of the indentation of a sphere against a hard flat 


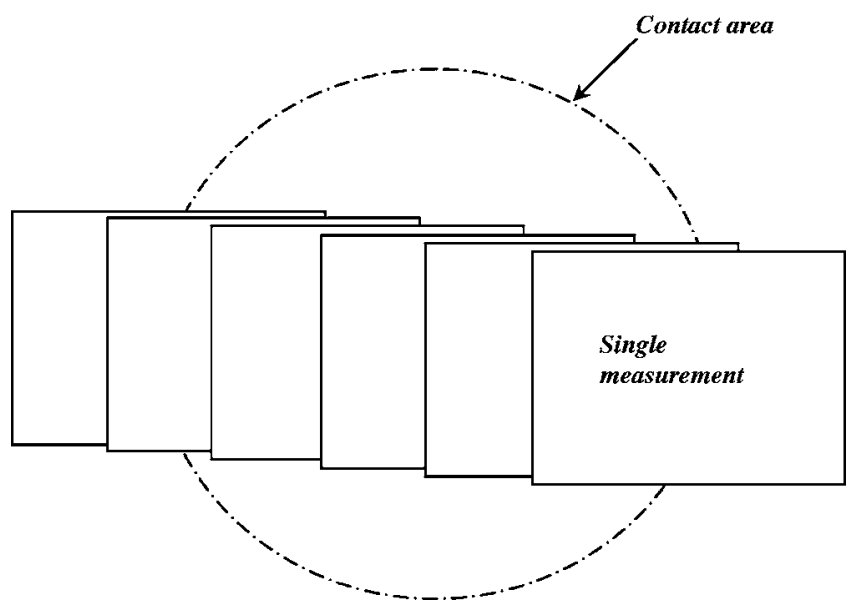

Fig. 2 Matching and stitching procedure

$a$ is the contact radius and $R$ is the initial radius of the copper sphere. This shows that the specimens used did not show a strain hardening effect.

In order to get the more accurate results experiments were also performed with another material. Aluminium spheres $(H$ $=0.28 \mathrm{GPa}, E=75.2 \mathrm{GPa}, \nu=0.345)$ with a diameter of $6 \mathrm{~mm}$ were used and the hard flat specimen used was $\mathrm{SiC}$. The results of the aluminium experiments, see the Appendix, are similar to the results of the copper specimens, therefore in the following the analysis is done for copper only.

2.2 Matching and Stitching. In most situations it is not possible to get an accurate or detailed image of a complete cross section of the contact area. For an accurate image of the cross section a high lateral resolution is needed, and as a result only small areas can be measured. To overcome this problem Sloetjes et al. $[15,16]$ developed a new measuring technique by matching and stitching a number of small but detailed images together from sequence measurements, as presented in Fig. 2.

The matching of two images can be defined as aligning or repositioning the overlapping part of two successive images. In order to get a detailed or wide image of a complete section across the contact area, the stitching process has to be performed. Several measurements are taken in the stitching process and each one having a certain overlap area with the previous one. For every stitching of the subsequence two images the mutual translation and rotation can be determined based on the overlapping area (matching process). Once all the images are matched, one large image is created as a complete of the stitching process. Figure 2 shows schematically the matching and stitching procedure used in the present experiments. The developed contact area from the plastic compression of the sphere is wider than one single measurement of the optical profiler, therefore, the matching and stitching procedure is needed to get the more precise measurement.

2.3 Experimental Details. Before doing any test, the spherical and flat specimens were cleaned with acetone and dried in air. To reduce the effect of friction, the contact region was lubricated. The load was applied to the sphere specimen for $30 \mathrm{~s}$ and then removed. Prior to measuring the fully plastic contact area, again the sphere was cleaned and dried.

An optical interference microscope was used to measure the plastic contact area. In this step the matching and stitching procedure was employed. A special holder, by which one is able to rotate and translate the sphere specimen, was designed to make the matching and stitching procedure easier. After taking all the images the matching and stitching calculation was done separately by a personal computer.

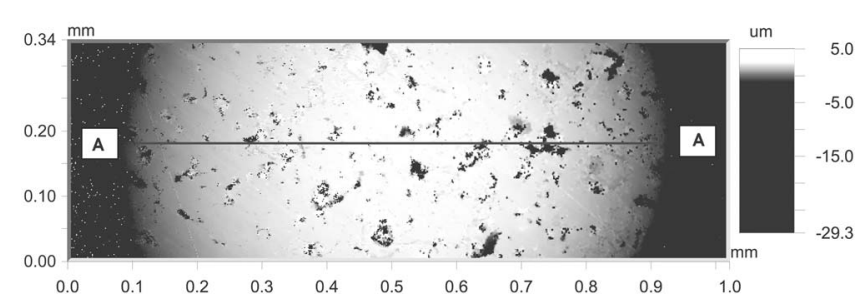

(a)

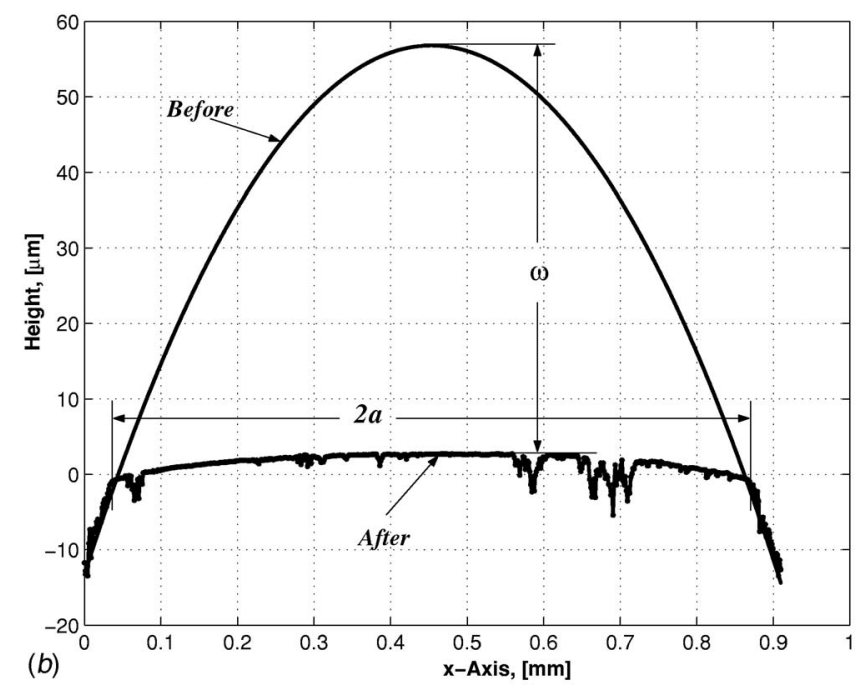

Fig. 3 (a) Matching and stitching of five images for a $490 \mathrm{~N}$ normal load. (b) A-A profile of (a).

\section{Results and Discussion}

As described above, to get results of a single experiment several steps must be followed. Eleven copper sphere specimens were used to perform the tests. Figure 3 shows a typical matching and stitching result of the plastic deformation of a sphere after compression. Five images have been matched and stitched to cover the total diameter of the plastic contact area at $P=490 \mathrm{~N}$. According to the JG model, the dimensionless normal load in this case was $P / P_{c-\mathrm{JG}}=1923$, far into the fully plastic regime. As can be seen in Fig. 3(a), the matching and stitching of the images results in an almost straight line, which implies that the translation and rotation specimen holder table works very well. Missing data was found in the images at the start and the end of the contact region. This is caused by the bad reflection of the surfaces, as can be seen more clearly in the profile plot in Fig. 3(b). At the edge of the contact the sudden change of the geometry results in a high slope. From this profile it can be seen that the effect of "piling up" and "sinking in" is less pronounced, as indicated by the almost coincide profiles between "before" and "after" compression at the edges of the contact; see Figs. 3(b) and 9 (later). These effects are introduced when the load is increased, however, the deviation between the measured maximum and minimum diameter of the contact area due to these effects is less than $3 \%$ in the present experiments.

The experimental results of the plastic contact area as a function of the contact interference, along with the theoretical model predictions of AF, CEB, and JG, are presented in Fig. 4. Here, the plastic contact area was measured by measuring the contact radius from the plastic deformation trace, as shown in Fig. 3(b). The method to measure the plastic interference was done by measuring the maximum difference between "before and "after" compression profile. Based on the deformed profile the undeformed (before) profile was calculated by the already known sphere radius and two points in the profile references, the first and the end images. As 


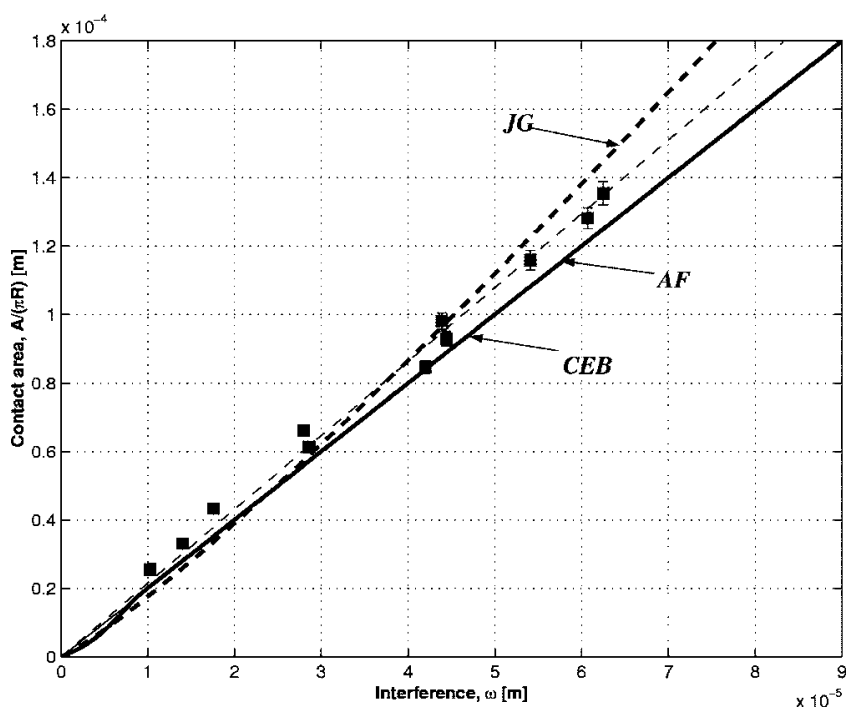

Fig. 4 Plastic contact area versus interference. $\square$ are experimental data.

can be seen, the experimental results agree well to the AF and CEB model. According to Eq. (3) for very small $\omega_{c-\mathrm{CEB}} / \omega$ (very high load), the AF and CEB model almost coincide. The measured plastic contact area is almost equal to $2 \pi R \omega$, as predicted by the AF model. The experimental results (the thin dashed line represents the best fit of the experimental data) show a slight higher slope than predicted by the AF model. This is because the measurement of the interference is based on the highest point of the deformed sphere; see Fig. 3(b). If the measurement of the interference is started from the datum of the contact area edge, then the experimental data become closer to the AF model. The plastic deformation is not perfectly as a truncation by a rigid flat but there is a slight crown at the center of the contact. The same phenomena have also been shown by the work of Johnson [17]. The crown is symmetrical around the center indicating that the pressure distribution was also symmetrical. The JG model predicts a smaller contact area than that of the AF and the CEB model for low loads, and the contact area increases more gradually as the load increases compared to the AF and the CEB model. The deviation of the JG model to the AF and the CEB model and the experimental results is larger for high loads.

The behavior of the mean contact pressure as a function of the plastic contact radius is plotted in Fig. 5. It shows that for all loading cases the mean contact pressure remains constant at a value of about $0.75 \mathrm{H}$. The present observations can be compared to previously published works. The work of Johnson [17] for instance, studied the deformation of the contact between two equal work-hardened copper spheres (radius $63.5 \mathrm{~mm}$ ). Results showed that the mean contact pressure in the fully plastic regime is constant for about $0.79 H$. The experimental results of Chaudhri [18] reported a constant value of the mean contact pressure of about $0.7 \mathrm{H}$ in the fully plastic regime in the case of the indentation of a copper sphere (radius $1.5 \mathrm{~mm}$ ) against a hard sapphire flat. These measurements compare very well with those made in the present observation.

The present experimental results, however, are lower and higher than that predicted by the AF model and the CEB model, respectively. A more quantitative comparison between the experimental

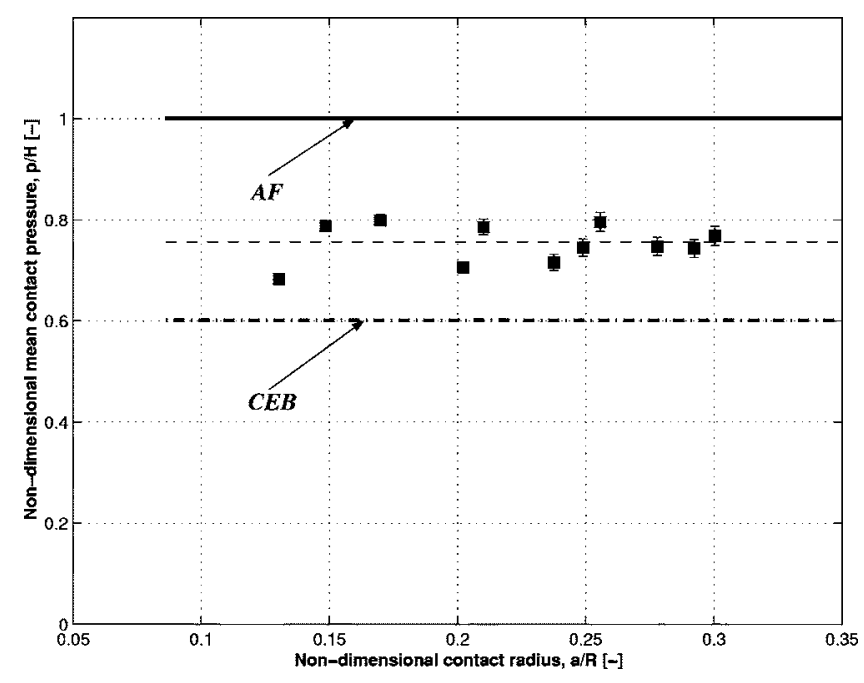

Fig. 5 Mean contact pressure against the nondimensional contact radius $a / R$. $\square$ are experimental data.

and the predicted mean contact pressure as a function of the contact area of the available models based on the experimental conditions as used in the present experiment is presented in Fig. 6. As can be seen, the theoretical model of JG predicts a higher mean contact pressure for the lower contact area (lower load) and underestimates the mean contact pressure for the higher contact area (higher load). The present experiment shows an almost constant mean contact pressure for the whole measured contact area. According to Tabor [19], as a first approximation, the fully plastic yielding of the indentation of a hard sphere against a deformable half-space occurs when the mean contact pressure $p$ equals

$$
p=c Y=c_{h} H
$$

where $c$ is nearly a constant and has a value of about 3 and $c_{h}$ is a hardness constant. If it is assumed that the hardness is equal to three times the yield strength $Y$ then $c_{h}$ has a value of about 1 . Interestingly, the AF model predicts fairly close to the experimental results when the mean contact pressure equal to $0.75 H$ (shown by the dashed line) or the $c_{h}$ value of Eq. (19) to be 0.75 . This

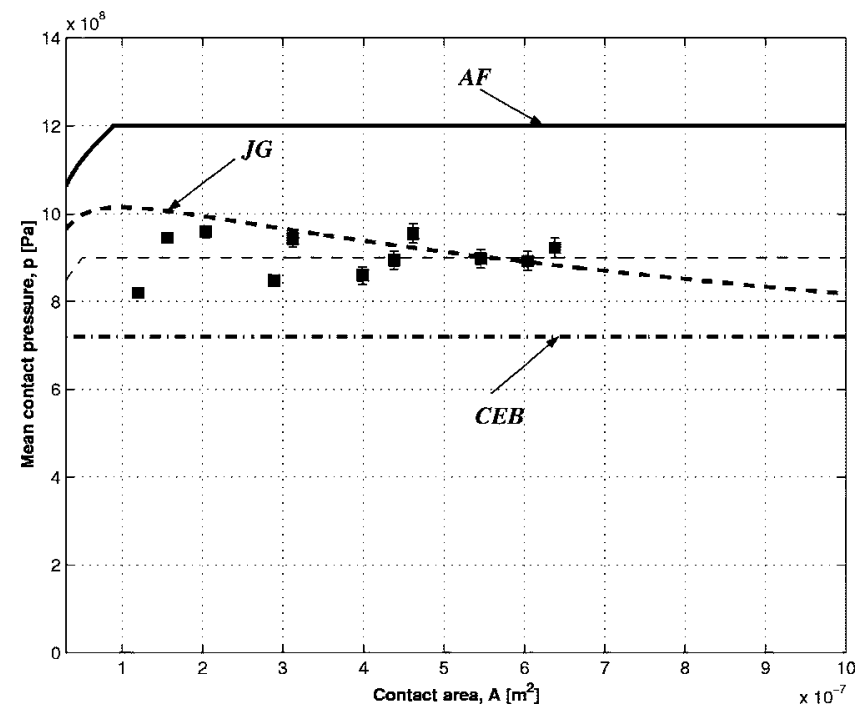

Fig. 6 Mean contact pressure versus contact area. $\square$ are experimental data. 

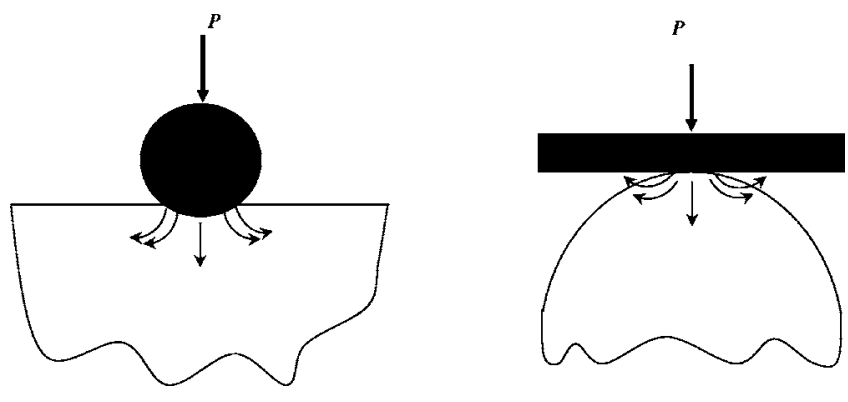

Fig. 7 Behavior of a hard sphere indenting a deformable halfspace and a hard flat indenting a deformable sphere

contradicts the most widely used value that is used for the fully plastic contact regime, i.e., the mean contact pressure is simply equal to its hardness value. Indentation experiments by Tabor [19], for instance, used a hard spherical indenter against a deformable half-space, but in the present experiment a deformable sphere is indented by a hard flat. Intuitively, for a very high load these two cases will pronounce a different behavior with respect to the deformation, as schematically shown in Fig. 7. In the first case the displaced material in the indented half-space is confined by the elastic-plastic deforming bulk of the half-space and the hard spherical indenter. This situation will produce a higher mean contact pressure and so increases the coefficient value $c_{h}$ in Eq. (19). But in the second case the displaced material of the deformable sphere is free to expand radially in the edge of the contact and the contact radius increases as the load increases.

Figure 8 presents the results of the contact area as a function of the normal load. Again, the present experimental results lie along the straight line for $p_{a}=0.75 H$ (dashed line) of the AF model. The CEB model overestimates the contact area as a function of the contact load that contradicts Fig. 4 when it is plotted as a function of the contact interference. This is caused by the mean contact pressure predicted by the CEB model that is lower than the actual one. The same phenomenon also occurs with the AF model where the mean contact pressure is predicted to be higher. Depending on the material properties the JG model predicts the contact area as a function of the load differently. However, the JG model tends to overestimate the contact area as the load increases. The difference will be more pronounced for higher loads. This overestimation is caused by the decrease of the mean contact pressure, as predicted by the JG model.

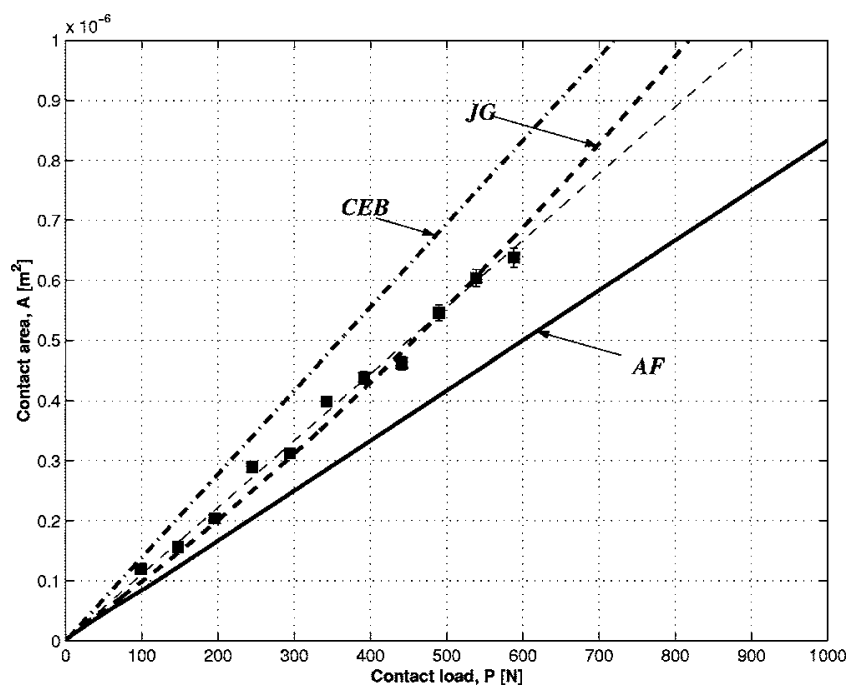

Fig. 8 Contact area as a function of normal load. $\square$ are experimental data.

\section{Conclusions}

The experimental investigation of the contact between a deformable sphere and a rigid flat was carried out to explore its behavior in the fully plastic contact regime for elastic-perfectly plastic material. The experimental results were compared with the published models in terms of the mean contact pressure, the contact area, and the contact load in order to make a comparison of the most frequently used models.

There are two main significant findings with respect to the present experiments. At first, the mean contact pressure was constant and has a value of about $0.75 \mathrm{H}$ for copper and $0.72 \mathrm{H}$ for aluminium, which is not confirmed by the proposed contact models. This suggests a constant value lower than 1 but higher than 0.6 for the hardness coefficient for the case of a deformable sphere in contact against a hard flat. Second, the work of AF, likewise CEB model for large interferences, is confirmed that the contact area is simply the truncation of the sphere by the hard flat for a fully plastic contact regime. A comparison of the present experimental results with the published asperity contact models showed substantial differences.

\section{Acknowledgment}

The financial support of SKF ERC B.V. Nieuwegein, The Netherlands is gratefully acknowledged.

\section{Nomenclature}

$$
\begin{aligned}
A & =\text { area of contact } \\
C & =\text { coefficient of critical yield stress } \\
E & =\text { Hertz elastic modulus } \\
E_{1,2} & =\text { Young's moduli } \\
H & =\text { hardness } \\
H_{G} & =\text { hardness geometric limit } \\
K & =\text { hardness factor } \\
P & =\text { contact load } \\
R & =\text { radius of the sphere } \\
Y & =\text { yield strength } \\
c_{h} & =\text { hardness constant } \\
p & =\text { mean contact pressure } \\
\nu_{1,2} & =\text { Poisson's ratio } \\
\omega & =\text { interference }
\end{aligned}
$$

\section{Subscripts}

$$
\begin{aligned}
\mathrm{AF} & =\text { Abbott-Firestone } \\
\mathrm{CEB} & =\text { Chang-Etsion-Bogy } \\
\mathrm{JG} & =\text { Jackson-Green }
\end{aligned}
$$

\section{Appendix: Aluminium Results}

Results of the contact between aluminium spheres against hard $\mathrm{SiC}$ flats are presented in Figs. 9-12. In the fully plastic contact regime, profile of the deformed spheres showed almost a flat following the shape of the flat indenter. Figure 9 shows the profile after indentation for a normal load of $206 \mathrm{~N}$. This typical form applies for all the experimental results. Therefore, the plastic contact area $(\mathrm{A}=2 \pi R \omega)$ is just the truncation of the sphere, as is shown in Fig. 10. For aluminium the mean contact pressure in the fully plastic contact regime in the present experiments is about $0.72 \mathrm{H}$; see Fig. 11. These results confirm the experimental work of Chaudhri [20], where the mean contact pressure in the fully plastic contact regime for elastic-plastic spheres with a diameter of $3 \mathrm{~mm}$ in contact with a sapphire flat is about $0.68 \mathrm{H}$. The plot of the contact area as a function of the load for the aluminium case can be seen in Fig. 12. 


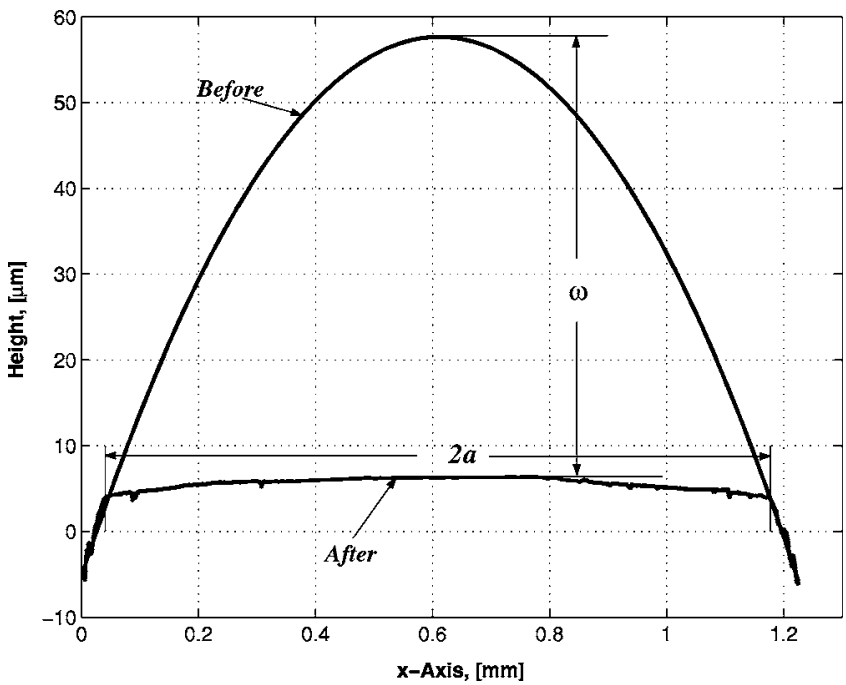

Fig. 9 Profile of the matching and stitching results for a $206 \mathrm{~N}$ normal load of an aluminium sphere

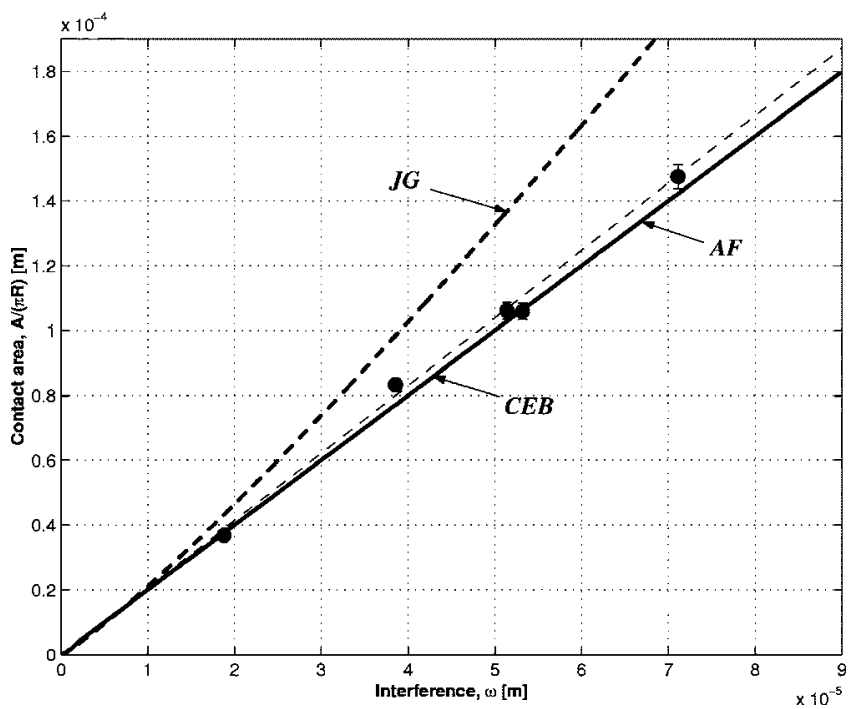

Fig. 10 Plastic contact area versus interference of aluminium spheres. are experimental data.

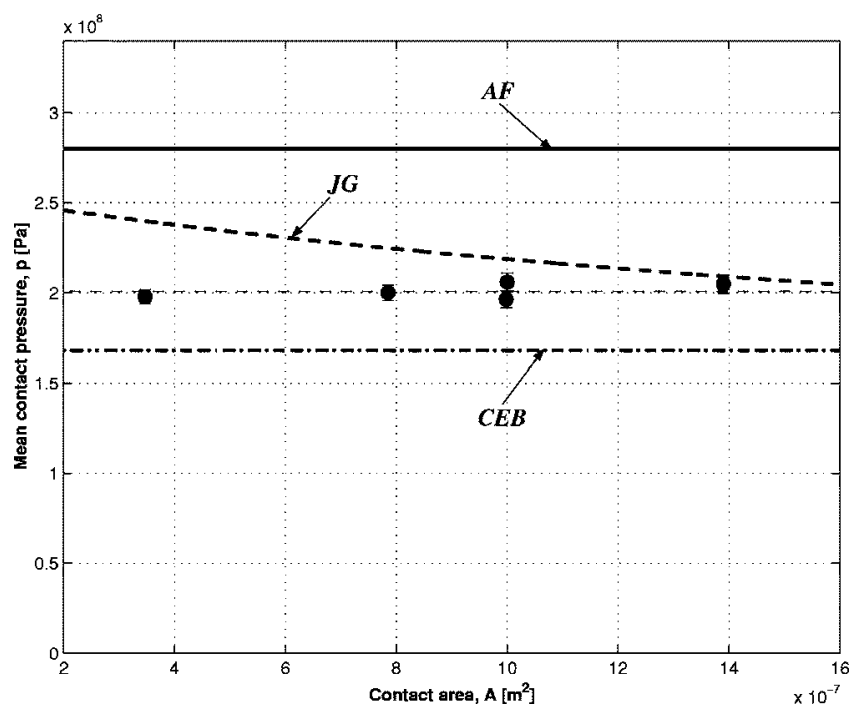

Fig. 11 Mean contact pressure versus contact area of aluminium spheres. $\bullet$ are experimental data.

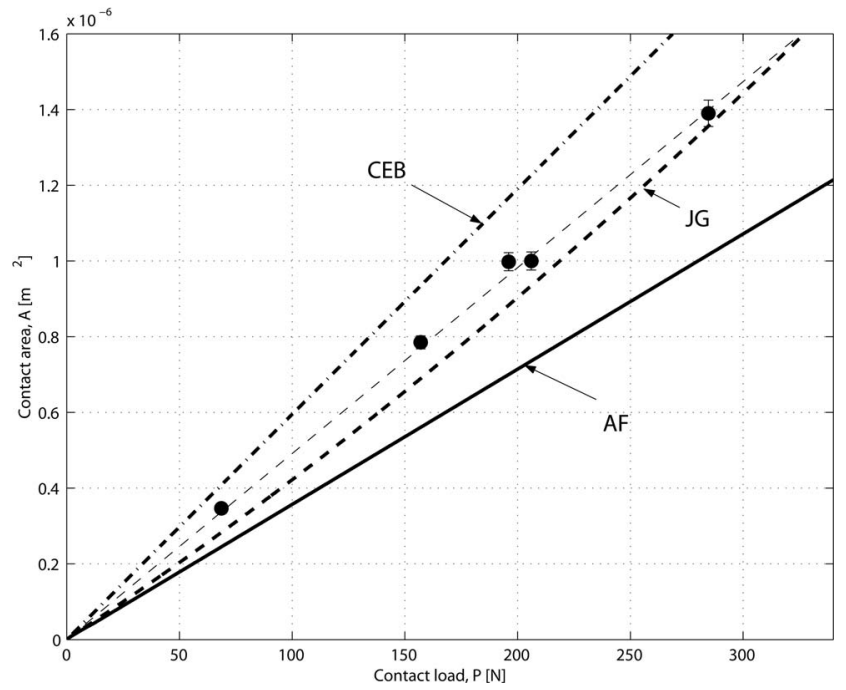

Fig. 12 Contact area as a function of the normal load of aluminium spheres. $\bullet$ are experimental data.

\section{References}

[1] Greenwood, J. A., and Williamson, J. B. P., 1966, "Contact of Nominally Flat Surfaces," Proc. R. Soc. London, Ser. A, 295, pp. 300-319.

[2] Abbott, E. J., and Firestone, F. A., 1933, "Specifying Surface Quality-A Method Based on Accurate Measurement and Comparison," Mech. Eng. (Am. Soc. Mech. Eng.), 55, p. 569.

[3] Chang, W. R., Etsion, I., and Bogy, D. B., 1987, "An Elastic-Plastic Model for the Contact of Rough Surfaces," ASME J. Tribol., 109, pp. 257-263.

[4] Horng, J. H., 1998, "An Elliptic Elastic-Plastic Asperity Microcontact Model for Rough Surface," ASME J. Tribol., 120, pp. 82-88.

[5] Zhao, Y., Maietta, D. M., and Chang, L., 2000, "An Asperity Microcontact Model Incorporating the Transition from Elastic Deformation to Fully Plastic Flow," ASME J. Tribol., 122, pp. 86-93.

[6] Jeng, Y. R., and Wang, P. Y., 2003, "An Elliptical Microcontact Model Considering Elastic, Elastoplastic, and Plastic Deformation," ASME J. Tribol., 125, pp. 232-240.

[7] Vu-Quoc, L., and Zhang, X., 1999, "An Elastoplastic contact ForceDisplacement Model in the Normal Direction: Displacement-Driven Version," Proc. R. Soc. London, Ser. A, 455, pp. 4013-4044.

[8] Vu-Quoc, L., Zhang, X., and Lesburg, L., 2000, "A Normal ForceDisplacement Model for Contacting Spheres Accounting for Plastic Deformation: Force-Driven Formulation," ASME J. Appl. Mech., 67, pp. 363-371.

[9] Mesarovic, S. D., and Fleck, N. A., 1999, "Spherical Indentation of ElasticPlastic Solids," Proc. R. Soc. London, Ser. A, 455, pp. 2707-2728.

[10] Mesarovic, S. D., and Fleck, N. A., 2000, "Frictionless Indentation of Dissimilar Elastic-Plastic Spheres," Int. J. Solids Struct., 37, pp. 7071-7091.

[11] Li, L.-Y., Wu, C.-Y., and Thornton, C., 2002, "A Theoretical Model for the Contact of Elastoplastic Bodies,” Proc. Inst. Mech. Eng., Part C: J. Mech. Eng. Sci., 216, pp. 421-431.

[12] Kogut, L., and Etsion, I., 2002, "Elastic-Plastic Contact Analysis of a Sphere and a Rigid Flat,” ASME J. Appl. Mech., 69, pp. 657-662.

[13] Jackson, R. L., and Green, I., 2003, “A Finite Element Study of Elasto-plastic Hemispherical Contact," Proceeding of 2003 STLE/ASME Joint Tribology Conference, 2003TRIB-268.

[14] Jackson, R. L., and Green, I., 2005, "A Finite Element Study of Elasto-Plastic Hemispherical Contact Against a Rigid Flat," ASME J. Tribol., 127, pp. 343354.

[15] Sloetjes, J. W., Schipper, D. J., Lugt, P. M., and Tripp, J. H., 2000, "The Determination of Changes in Surface Topography Using Image Processing Techniques," in Proceedings of the International Tribology Conference, Nagasaki.

[16] Sloetjes, J. W., Tasan, Y. C., De Rooij, M. B., and Schipper, D. J., 2002, "Algorithm for Determining Changes in Micro-Geometry Using Image Processing Techniques," in Proceedings of the 2nd Asia International Conference on Tribology, Asiatrib '02, Cheju Island, Korea.

[17] Johnson, K. L., 1968, "An Experimental Determination of the Contact Stresses Between Plastically Deformed Cylinders and Spheres," Engineering Plasticity, Cambridge University Press, Cambridge, pp. 341-361.

[18] Chaudhri, M. M., 1987, "The Plastic Deformation of Single Asperities by Hard Flats," Proc. Inst. Mech. Eng., 158, pp. 1003-1012.

[19] Tabor, D., 1951, The Hardness of Metals, Clarendon Press, Oxford, UK

[20] Chaudhri, M. M., Hutchings, I. M., and Makin, P. L., 1984, "Plastic Compression of Spheres," Philos. Mag. A, 49, pp. 493-503. 